Estudios sobre armas antiguas, arte militar y vida cultural en oriente y occidente XXX (2010), pp. 79-98 ISSN : 0436-029X

doi: 10.3989/gladius.2010.0004

\title{
LAS ARMADURAS ROMANAS EN HISPANIA: PROTECTORES CORPORALES PARA LA INFANTERÍA Y LA CABALLERÍA
}

\author{
ROMAN ARM OUR IN HISPANIA: BODY PROTECTION \\ FOR INFANTRY AND CAVALRY
}

POR

Joaquín A urrecoechea*

\section{Resumen - A bstract - Résumé}

El propósito del presente trabajo es el estudio de las armaduras romanas halladas en Hispania. Tras la revisión de las fuentes arqueológicas, se propone una visión de síntesis sobre estas piezas en la península ibérica, donde se documentan todas las variantes de armaduras conocidas. L os yacimientos hispanos han proporcionado la cronología final más avanzada de todo el Imperio, respecto a las armaduras segmentadas y los pectorales repujados.

The main subject of this paper is the study of roman armours found in $\mathrm{H}$ ispania. A fter a revision of the archaeological evidence, the article suggests a survey in Iberian Peninsula. In that territory, all the recognizable categories of roman armours are attested. Sites from Hispania offers the most advanced late chronology in the whole E mpire, regarding with lorica segmentata and embossed breastplate armours.

Ce travail étudie les armures romaines trouvées en Hispania. A près une révision des sources archéologiques disponibles, il propose un bilan de ce type de pièces pour l'ensemble de la péninsule l bérique, où toutes les catégories d'armures sont attestées. Les sites hispaniques fournissent notamment les attestations les plus anciennes pour la lorica segmentata et pour les pectoraux décorés au repoussé.

Palabras Clave - Keywords - M ots clés

A rmadura romana; Lorica segmentata; Lorica hamata; Lorica squamata; M anica; Grebas; Pectorales; Hispania.

Roman armour; Lorica segmentata; Lorica hamata; Lorica squamata; Manica; Roman Greaves; Roman breastplate; Hispania.

Armure romaine; Lorica segmentata; Lorica hamata; Lorica squamata; M anica; Jambières; Pectoraux; Hispanie.

\section{INTRODUCCIÓN}

Los estudios sobre armaduras se han dinamizado en los últimos años gracias al empuje dado por los trabajos de Bishop y colaboradores. El interés suscitado por sus publicaciones ha originado la aparición de un auténtico elenco de piezas hasta ahora inéditas, así como la reinterpretación científica de viejos hallazgos. Entre los últimos descubrimientos cabe citar los de Zeugma (Turquía) (Feugère, 2006) y Carlisle (Inglaterra) (M cCarthy, Bishop y Richardson,

* Paseo M arítimo Ciudad de M elilla, 17, 6-E (29016), M álaga-SPA IN . E-mail: jaurreco@uma.es 
2001), debido al volumen e importancia de los restos. No obstante, aún existen significativos desequilibrios respecto a nuestro conocimiento sobre los distintos tipos de armaduras, pues priman las publicaciones sobre categorías concretas, como las lorigas segmentadas, frente a la escasez de trabajos sobre otras clases de protectores. A sí mismo, nuestra percepción geográfica se centra en los descubrimientos de la Pars O ccidentalis del Imperio, siendo la parte oriental peor conocida.

Hispania se ha incorporado recientemente al panorama internacional, salvando la distancia que la separaba de otras provincias del Imperio. No obstante, nuestro avance ha estado centrado en las corazas segmentadas, campo en el que hemos pasado de ser unos desconocidos a ser un punto de referencia, debido a la abundancia de piezas y los interrogantes cronológicos que plantean ${ }^{1}$. Podemos definir el estado actual de la investigación por la falta de una visión de conjunto, situación que compartimos con otras zonas mejor conocidas, como Britania o Germania, donde tampoco existen síntesis semejantes ${ }^{2}$. Con el presente trabajo queremos abrir una nueva vía de investigación que fomente los estudios espaciales para definir variantes regionales, aportando los materiales de la Hispania romana ${ }^{3}$.

\section{LAS ARMADURAS ROMANAS}

La armadura es un arma primordial en cualquier ejército, estando definida por tres aspectos fundamental es: garantizar la protección ante las armas de los enemigos, poseer flexibilidad suficiente como para permitir la necesaria libertad de movimiento, y ser lo suficientemente ligera. Dichas exigencias influyeron decisivamente en la evolución de la coraza romana, pues ésta es el resultado del compromiso entre movilidad y protección. El perfeccionamiento de las armaduras romanas estuvo marcado también por dos factores principales: determinismo técnico y adecuación a los escenarios de guerra. G racias a los avances técnicos se consiguieron materiales más ligeros y resistentes, mientras que el contacto con las fuerzas enemigas obligó a constantes cambios en función del equipo militar que éstas empleaban. A sí, de griegos, partos, sármatas y celtas tomaron prestados una serie de modelos, como la coraza de escamas o la cota de malla, hasta alcanzar una creación total mente original, la lorica segmentata. Estas tres categorías mencionadas, junto a la coraza musculada, configuran los principales tipos usados por el ejército romano.

\section{LAS ARMADURAS SEGMENTADAS}

La lorica segmentata es el prototipo mejor conocido de todos los empleados por el ejército romano. Se trata de una coraza formada por láminas de hierro articuladas. En su interior cuenta

1 A estas piezas hemos dedicado varios estudios recientes (A urrecoechea, 2006, 2007 y A urrecoechea et alii 2008), por lo que serán tratadas aquí de forma sucinta. Por ello omitiremos las referencias a unidades estratigráficas donde han aparecido, dato que sólo ofreceremos en el caso de piezas inéditas o singulares.

2 A Robinson (1975) debemos un trabajo general sobre el Imperio, si bien se limitó a recoger los ejemplares más notables, sin establecer conclusiones sobre provincias concretas.

3 Q ueremos agradecer las facilidades ofrecidas para la consul ta de materiales leoneses a Victorino García M arcos, Fernando M uñoz Villarejo y Carlos Ál varez Ordás. También queremos expresar nuestro reconocimiento a Rosario García Rozas, por el acceso y documentación gráfica de las piezas depositadas en el M useo de Zamora; así como a Santiago Carretero, Victoria Romero y M anuel Caamaño, por su gentileza al poner a nuestra disposición piezas en estudio de sus respectivas excavaciones. Finalmente queremos manifestar nuestra deuda con Á ngel M orillo Cerdán, quien con su ayuda y consejo hizo posible esta investigación. 
con tiras de cuero que unen las distintas placas, mientras que externamente posee una serie de accesorios de cobre/bronce que sirven para articular o abrochar las distintas secciones de la armadura.

Las peculiaridades que presentan ha llevado a postular que este tipo de defensas eran características de determinadas legiones, sobre todo de aquellas que tenían que adaptar sus tácticas de guerra para combatir a enemigos que luchaban al estilo de los celtas, lo que requería una mayor protección en la cabeza y los hombros de los soldados de infantería que se situaban en las primeras líneas de batalla (Bishop y Coulston, 1993: 209)4. Dicha teoría queda desmentida, a nuestro juicio, por la enorme dispersión geográfica de las loricae segmentatae y su continuado uso durante toda la época imperial. Casi todas las armaduras romanas, y no solo las segmentadas, cuentan con una mayor protección y deterioro en la zona de los hombros, como más adelante veremos al hablar de las cotas de malla. Salvaguardar el punto débil de los hombros era de vital importancia cuando se combatía con enemigos que usaban espadas largas, puesto que cuando el soldado se resguardaba tras su escudo era sólo vulnerable en los hombros y la cabeza. Si tenemos en cuenta que los cascos romanos se diseñaron para proteger la cabeza desviando los golpes hacia los hombros, nos daremos cuenta del sentido que tenía sobreproteger esa zona. Algunos investigadores abogan por la mayor utilidad en los combates de la armadura segmentada frente a otro tipo de corazas, pues ésta absorbía mejor los gol pes (Williams, 1977: 77). Precisamente la curvatura de las placas de la lorica segmentata, que se adaptaba al torso del soldado, favorecía el desvío de los golpes directos sobre ella, lo que potenciaba su efectividad. O tra ventaja es que contaba con un peso menor que otros protectores, como la lorica hamata.

Tipológicamente se han definido tres arquetipos principales: «K alkriese», «Corbridge» y «N ewstead»; más una categoría híbrida («A lba Iulia») (Bishop, 2002). L a estructura morfológica de estas variantes es muy similar, por lo que los distintos tipos se definen básicamente por los el ementos funcionales o decorativos en cobre/bronce que presentan en su cara externa. A simismo, la evolución temporal que sufrió cada categoría concreta tiene su exponente más relevante en los diferentes perfiles que caracterizaron a estos apliques.

\section{- Armaduras del tipo «Kalkriese»}

El protector segmentado más antiguo de toda la serie es el tipo «K alkriese» (Bishop, 2002: 23-30). Dicha categoría era desconocida hasta hace poco, siendo aún provisional es las reconstrucciones de su estructura y las variantes que presenta. Entre los vestigios más antiguos se encuentran los hallados en Dangstetten (Alemania), datados al rededor del 9 a.C. (Fingerlin, 1986: 136, lám. 371); mientras que algunos de los más modernos están relacionados con la conquista de B ritania, con exponentes en Chichester y Waddon Hill (D own, 1989: fig. 27).

Este tipo de armaduras está escasamente representado en la península ibérica, reduciéndose su presencia a una serie de bisagras. L os perfiles de las bisagras «K alkriese» son bastante simples, pudiendo presentar un par de lóbulos dentro de la estructura rectangular de la pieza, o contar con un remate apuntado. El ejemplar más antiguo está asociado al campamento de la legio X gemina en A storga (L eón) (fig. 1, 1), descubierto en las excavaciones de la Plaza de Calvo Sotelo, 10 (A urrecoechea, 2007: 159, fig. 3, 1) y fechado en época augusteo-tiberiana 5 . Se trata de un gozne apuntado perteneciente a la categoría «F.i» de la tipología de Thomas

4 También habría que tener en cuenta las peculiaridades de cada zona, así la lorica segmentata parece haber sido menos adecuada a los escenarios de guerra orientales (M enéndez, 2006: 105-117).

5 La datación de los contextos arqueológicos leoneses que ofrecemos está basada en las investigaciones desarrolladas por Victorino García M arcos y Ángel M orillo (M orillo y García, 2006) enmarcadas dentro del proyecto del M inisterio 
(2003: 62-63), que cuenta con buenos paral elos en los yacimientos augusteos del limes germano. A dscritos a la categoría «F.ii» de Thomas son dos bisagras bilobuladas de I ruña y A storga. La primera de ellas, hallada en Veleia (I ruña de Oca, Álava) (Filloy y Gil, 2000: no 353), parece estar datada en la segunda mitad del siglo I; mientras que la pieza asturicense apareció vinculada a las obras de cimentación de las Termas M enores (c/ Padre Blanco, 7/11) (fig. 1, 2), fechándose en las postrimerías de la primera centuria (A urrecoechea, 2007: 159, fig. 3, 2). Estos últimos ejemplares pertenecen al grupo de los accesorios tardíos dentro de esta categoría de armaduras, pues las piezas bilobuladas no aparecen ni en el yacimiento de K alkriese (A lemania) ni en los campamentos del limes de principios del siglo I. Paral elos casi exactos han sido descubiertos en Chichester (Inglaterra) (40/50 d.C) (Down, 1989: fig. 27.5, 80) y Kaiseraugst (Suiza) (70/75 d.C.) (Thomas, 2003: 63, fig. 46, 3).

\section{-Armaduras del tipo «Corbridge»}

Son las más mejor conocidas de todas las armaduras segmentadas, debido a la abundancia de hallazgos y su amplia dispersión geográfica. Robinson (1975: 177-180) distinguió tres subtipos diferentes, si bien discernir unas variantes de otras cuando sólo se hallan meros fragmentos es una labor imposible. Por ello cobran importancia nuevamente los complementos en cobre/bronce de estos protectores, verdaderas señas de identidad que los definen. Los elementos más característicos son los discos repujados, decorados con pétalos radiales o círculos concéntricos, y las charnelas trilobuladas. J unto a ellos, las presillas caladas que hacen de pieza «hembra» para alojar los ganchos de la faja, si bien su similitud con otros objetos de la vida cotidiana hacen difícil reconocerlas cuando aparecen aisladas. M enos representativos son los mencionados ganchos, los broches con hebillas y las placas charneladas para correas, pues todas ellas están presentes tanto en el tipo «Corbridge» como en el «N ewstead».

Respecto a su origen, se ha señalado su evolución de la categoría «K alkriese» en un momento indeterminado de la etapa tiberio-claudia (B ishop, 1998: 11), estando ampliamente representadas estas armaduras entre los contingentes que invadieron B ritania. Durante los años central es del Imperio se prodigan en casi todos los establecimientos legionarios de Occidente, siendo aún imprecisa la fecha final de utilización. Hasta ahora se había señalado su presencia en el Waffenmagazin de Carnuntum (A ustria) como indicativa de un postrero uso hacia la mitad del siglo II (Bishop, 1998: 12), aunque tal cronología ha sido rebatida por los recientes hallazgos vinculados a los yacimientos de León (A urrecoechea, 2007: 177-179), Ios cuales demuestran que el tipo perduró mucho más de lo que se podía prever.

L os primeros testimonios de armaduras «Corbridge» en H ispania están vinculados al campamento de la legio VI en L eón, siendo la evidencia más antigua de su utilización el gancho documentado en un vertedero tibero-neroniano de La Palomera (A urrecoechea, 2007: 161, fig. 3, 3). Dicha cronología le convierte en una de las primeras evidencias del uso de estas lorigas en el I mperio, equivalente a los ejemplares atestiguados durante la conquista de B ritania. Prácticamente coetáneos, pues se datan en época claudio-neroniana, son los importantes restos de La Praviana, vinculados a un taller de fundición (A urrecoechea, 2006: 309-334; 2007: 162, fig. 3, 4-6 y fig. 8, 2-3). Se localizaron más de 20 grupos diferentes de flejes de hierro, entre los que podemos identificar varias láminas de hombreras y una plancha correspondiente a la faja (fig. 3, 3). I gual mente pertenecientes al asentamiento de la legio VI en León son tres flejes

de Educación y Ciencia (HUM 2006-00534): «Campamentos romanos en Hispania: análisis diacrónico de las estructuras defensivas». 
descubiertos en las excavaciones de la calle Plegarias c/v Ramiro III, asociados al nivel de derrumbe de un taller de forja, fechado en época flavia (A urrecoechea, 2006: 315).

En el valle del Ebro también se tiene constancia temprana de estas corazas, como podemos comprobar gracias a una hebilla documentada en C elsa (Zaragoza), datada en la primera mitad del siglo I (B eltrán, 1998: 105, no 12).

Continuando con el fuerte leonés, pero asociados al periodo de la legio VII, son un nutrido grupo de materiales. Desde el punto de vista cuantitativo destacan los vestigios hallados en otro taller de fundición, documentado en la Plaza del Conde L una (A urrecoechea, 2006: 309334, fig. 6-9; 2007: 162, figura 4, 1-8). Es reseñable que en dicho depósito, fechado en época tan avanzada como el último cuarto del siglo III, convivan restos de corazas «Corbridge» $y$ «N ewstead». Entre los materiales encontrados podemos citar bisagras trilobuladas, discos repujados con decoración vegetal a base de pétalos, placas charneladas, etc. (Figura 1: 4-7 y 9-11). Otro nutrido el enco de piezas procede del nivel de derrumbe de un almacén en torno a patio localizado en Puerta Castillo-Santa M arina, datado entre el 250-275 d.C. (A urrecoechea, 2007: 165-166, 173-175, fig. 5, 1-4). Se trata de los flejes pertenecientes a las láminas ventrales de una faja y un fragmento de peto de una armadura «Corbridge-A ».

Otras piezas del área leonesa están vinculadas al campamento de la legio X gemina en Asturica Augusta, entre ellas las aparecidas en las excavaciones de las fossae fastigatae, donde entre otros elementos se localizaron ganchos de lorica segmentata ${ }^{6}$. Avanzando en el tiempo, cuando A storga deja de ser la sede de la mencionada legión, el enclave vuelve a ofrecernos testimonios de armaduras «C orbridge» gracias a una bisagra hallada en la Plaza de Calvo Sotelo, 10 (A urrecoechea, 2007: 165, fig. 4, 9). Este último ejemplar, (Figura 1: 3), datado en el tránsito entre el siglo tercero y el cuarto, confirma la pervivencia de nuestras corazas hasta los inicios del Bajoimperio, pues debido a la cronología de la presencia militar en el yacimiento no podemos considerar estos restos como residuales de una época anterior (A urrecoechea, 2007: 177-179).

Para terminar de completar el panorama referido a estas piezas, sería obligado mencionar los descubrimientos realizados fuera del área leonesa, si bien la mayoría tienen un valor meramente testimonial, pues la información que poseemos es muy escasa. De Herrera de Pisuerga (Palencia) proceden bisagras y ganchos publicados sin alusión al contexto arqueológico (García, 1999: 14, fig. 1, 4; Fernández, 1994: fig. 2, 9. Pérez, 1996: fig. 5). También ignoramos el contexto en el que apareció la hebilla inédita encontrada en Rosinos de Vidriales (Zamora) (Figura 3,4$)^{7}$.

\section{- Armaduras del tipo «Newstead»}

L as armaduras «N ewstead» constituyen el modelo que culmina la evolución de las corazas segmentadas. Para Bishop (2002: 49) su temporalidad abarcaría desde la primera mitad del siglo II hasta mediados de la tercera centuria, aunque nuevamente tal cronología debe revisarse a tenor de los hallazgos leoneses, los cuales demuestran que perduraron hasta el Tardo I mperio (A urrecoechea, 2007: 170-71). Una de las características principales que define a las lorigas «N ewstead» es el modo de abrochar los flejes de la faja, mediante anillas de cobre/bronce que se hacen pasar por sendos orificios rectangulares enmarcados por placas también de bronce.

6 A unque no hemos tenido acceso a dichas piezas conocemos su existencia al mencionarse en algunos trabajos (González y Vidal, 2004: 207-218).

7 La pieza fue dada a conocer en el póster titulado «M ilitaria from Roman Forts in Petavonium (prov. Zamora, Spain)», presentado por Carretero, S. y Romero, M. V., en International Roman M ilitary Equipment Conference (ROMEC), cel ebrada en Vindonissa/Widish-B rugg (Suiza) en 2001, si bien no fue publicada. 
La presión ejercida sobre tal es anillas durante los movimientos del soldado prodigaba la rotura de las mismas, causa que justifica su abundancia en el registro arqueológico. Otro elemento específico son las bisagras lobuladas de gran tamaño, cuyos extremos son apuntados. R especto a los discos que las decoran, éstos son lisos y no repujados. Finalmente, otro rasgo propio es el enfundado de la parte baja del faldón con tiras de cobre/bronce dobladas en forma de «V».

Centrándonos en los hallazgos hispanos, estas armaduras se incorporan a la impedimenta de los soldados de la legio VII asentados en L eón desde sus primeros momentos de vida, como confirma la anilla del Edificio B otines fechada durante la primera mitad del siglo II (A urrecoechea, 2007: 169, fig. 7, 2). No obstante, el grueso de los materiales procede del mencionado taller localizado en la Plaza del Conde Luna, datándose a finales de la tercera centuria (A urrecoechea, 2006, 317-328; 2007: 169-170, fig. 6). Sobresalen, desde el punto de vista numérico, la docena de anillas adscritas al modelo «l.iii" de Thomas (2003: 109-113). También son dignas de mencionar tres bisagras lobuladas del tipo «F.viii" de Thomas (2003: 62-85); así como las fundas para faldón en su modalidad lisa y decorada (Figura 1: 8, 12-15 y Figura 3, 5).

Continuando con el fuerte de la legio VII en León, el resto de los ejemplares están asociados al periodo bajoimperial. De finales del siglo III a principios del IV es una anilla documentada en Puerta Obispo (A urrecoechea, 2007: 170, fig. 7, 5). En la cuarta centuria se fechan la anilla y la bisagra aparecidas en el vertedero ubicado en Santa M arina con San A Ibito (A urrecoechea, 2007: fig. 7, 1 y 3). También de los albores del siglo IV son un par de anillas inéditas descubiertas en las excavaciones de la c/ Cid c/v Ordoño (U .E. 13) (fig. 3, 6). A unque la cronología más reciente, que cae ya en pleno siglo $\mathrm{V}$, nos la ofrece otra anilla encontrada igualmente en Puerta Obispo (A urrecoechea, 2007: fig. 7, 4).

En el entorno próximo a Legio contamos con otras evidencias que confirman la utilización de lorigas «Newstead» en el mismo periodo. L as excavaciones del edificio central de Puente Castro (L eón) han deparado restos de anillas y ganchos, fechados entre la segunda mitad del siglo II y la primera mitad de la tercera centuria (A urrecoechea, 2007: 170, fig. 7, 6-7). De L ancia (León) procede otra anilla (A urrecoechea, 2007: fig. 7, 8). A storga ha proporcionado diversos elementos cuya datación discurre entre el último tercio del siglo III y comienzos de la cuarta centuria. Entre ellos, una funda para faldón localizada en la c/ La Cruz 20-24, un gancho descubierto en las termas de Padre B lanco y otro gancho proveniente de la Plaza Calvo Sotelo, 10 (A urrecoechea, 2007: fig. 7, 9-11).

Fuera del área geográfica leonesa apenas conocemos testimonios atribuibles a armaduras «Newstead»" ${ }^{8}$, salvo una anilla del campamento de Herrera de Pisuerga. Desconocemos su marco arqueológico (Fernández, 1994: fig. 2, 6.).

\section{ARMADURAS DE ESCAMAS}

L a lorica squa mata posiblemente sea uno de los tipos de armadura más antiguo usados por el hombre (Peterson, 1992: 42). Su estructura se compone de una serie de láminas, realizadas en hierro o cobre/bronce, imbricadas mediante ligaduras de alambre, cosiéndose después a una prenda de tela fuerte. A grandes rasgos podemos diferenciar dos tipos distintos de corazas. El modelo flexible fue el más usado durante todo el I mperio Romano, estando formado por láminas pequeñas que solo se unían horizontalmente. O tra modalidad fue la semirrígida, popularizada a

8 Gil (1997: 553) menciona «3 elementos de fijación para lorica segmentata» de Iruña, sin que concrete el tipo ni ilustre los ejemplares, por lo que no podemos asociarlos a ninguna de las categorías vistas. 
partir de la época antonina, y que consistía en escamas alargadas unidas entre sí por sus cuatro lados. Dicha disposición limitaba los movimientos del soldado en el plano vertical.

El tamaño de las láminas y su morfología abarcan un amplio rango de perfiles y secciones, desde las más comunes con puntas redondeadas hasta las de remate puntiagudo (Robinson, 1975: 154). L as escamas fueron habitualmente recubiertas de estaño, acabado que no sólo tenía un propósito estético sino también práctico, pues retardaba la corrosión del metal y evitaba que se pudrieran las puntadas que cosían la armadura al forro de tela.

Sobre su calidad defensiva, muchos autores la consideran inferior a la cota de malla, pues no era ni tan fuerte ni tan flexible (Simkins, 1994a: 15). Su popularidad en época romana se debió probablemente a otros factores, ya que eran más fáciles de fabricar y reparar que otras Ioricae. L os experimentos de M assey (1994: 56) sobre reconstrucciones modernas demuestran que, cuando las escamas estaban ligadas con al ambre enrollado, ninguno de los tipos de flecha contemporáneos podía penetrarla, si bien las escamas resultaban sensiblemente deformadas por el choque. Por tanto, la armadura impedía que el proyectil alcanzara el cuerpo, pero el impacto causaba traumatismos y hemorragia interna a la persona que se protegía con ella.

En Hispania se han encontrado diversos restos de estas piezas, principal mente en forma de escamas sueltas, todos ellos inéditos o publicados someramente. El testimonio más antiguo es un fragmento de lámina de cobre/bronce aparecida en León, concretamente en Santa M arina (U.E. 4070) (fig. 2, 5). L ocalizada en un nivel correspondiente a la segunda fase del campamento de la legio VI, se fecha en época tibero-neroniana. También de León, pero asociadas al taller de la legio VII datado a finales de la tercera centuria, son dos escamas suel tas fabricadas en cobre/bronce y un bloque unido aún por sus eslabones, hallados en la Plaza del Conde L una (U.E. 107) (Figura 2, 4)9.

Siguiendo con el entorno leonés, de Puente Castro procede un conjunto de escamas que aparecieron formando un bloque, el cual se disgregó durante las tareas de extracción (fig. 2, 6). Se localizaron en un potente estrato datado entre la segunda mitad del siglo II y la primera mitad del siglo III ${ }^{10}$. L as láminas de cobre/bronce debieron estar unidas a un fragmento de tela, a tenor de los indicios conservados, siendo probable que estuvieran estañadas en su superficie exterior. L as escamas estaban unidas sólo horizontalmente, mediante al ambres que atraviesan los orificios de las placas para formar una lazada simple, siendo el grosor de dicho alambre de apenas $1 \mathrm{~mm}$ de espesor.

Pertenecientes al campamento de la cohors I Celtiberorum en Cidadela (A Coruña) son también una serie de vestigios, hallados en el nivel de relleno originado tras abandonar el establecimiento militar, para el que se utilizaron tierras sacadas del propio campamento y de la cannaba, cuyos materiales arqueológicos han de fecharse a finales del siglo II y en especial en el siglo $\mathrm{III}^{11}$. Se trata de siete fragmentos de láminas, al gunas de las cuales aún se encuentran unidas por alambres ${ }^{12}$, correspondientes a los modelos «iv» y «vi» de G roller (1901: lám. 445).

9 Estas piezas no fueron incluidas en la publicación sobre dicho taller (A urrecoechea, 2006), pues pasaron inadvertidas entre el metal informe, siendo la restauradora M yriam Hernández Valverde del M useo de L eón quien posteriormente nos informó de su existencia, agradeciéndole desde aquí su amabilidad y profesionalidad.

10 Del conjunto de materiales que acompañaban a estas escamas cabe destacar la abundancia de las piezas metálicas, entre las que ya se han publicado las correspondientes al instrumental médico (Álvarez, Rodríguez y M artínez, 2000/01: 141-158).

11 A gradecemos a D. C aamaño G esto el acceso a los materiales, así como la interpretación del contexto en el que se insertan estas piezas. El entorno arqueológico está relacionado con el descrito a tenor del estudio de un ara que ya ha sido publicada (Caamaño, 1983: 61-71).

12 L as piezas fueron dadas a conocer en la exposición «Ciudad y Torre. Roma y la llustración en L a Coruña (Estación M arítima, 27 septiembre - 10 de noviembre de 1991)», publicándose una breve reseña de las mismas en el catálogo del 
En Herrera de Pisuerga se atestiguan un fragmento de escama correspondiente a una lorica semirrígida (García, 1999: 14, no 1), así como diversas escamas adscritas a la categoría «V» de Groller (1901: lám. 445), conservándose algunas de ellas aún unidas (García, 1999: 14, nos. 2 y 3$)$.

Los vestigios de lorica squamata en $\mathrm{H}$ ispania cubren una amplia gama de tipos, documentándose numerosas variantes de escamas, a pesar de la escasez de testimonios. También podemos afirmar que aquí se usaron los dos modelos principal es de estas corazas: el semirrígido y el flexible. U na característica que unifica a los hallazgos hispanos es que ninguno de ellos está vinculado claramente con el proceso de reciclaje del metal, a diferencia de lo que ocurre con la mayoría de las armaduras segmentadas encontradas en nuestra provincia, las cuales habían sido previamente desmanteladas para ser recicladas. Si las piezas hispanas hubieran sido productos de desecho preparados para el reciclaje, las escamas no conservarían los eslabones de enlace y probablemente habrían sido dobladas para formar una pieza compacta de metal más idónea para ser fundida, tal y como se constata en Piercebridge (Inglaterra) ${ }^{13}$.

\section{COTAS DE MALLA}

El origen de la lorica hamata esta ligado al mundo celta, de donde se habría transmitido a la metalistería romana (Varrón, D e Lingua Latina 5.116). L a armadura consiste en una serie de anillas encadenadas, de modo que cada una de ellas está unida a otras cuatro, dos correspondientes a la fila superior y dos a la inferior. Dichas anillas se confeccionaban en aleación cobre/bronce 0 en hierro, siendo su tamaño sorprendentemente pequeño, pues algunas apenas alcanzan los $3 \mathrm{~mm}$ de diámetro (Simkins, 1994b: 18). El tipo más habitual poseía mangas cortas o carecía de ellas, siendo mucho más rara la manga larga. L os hombros eran objeto de una especial protección, reforzando su defensa mediante unas hombreras abrochadas al frente de la camisa gracias a un doble gancho articulado que descansa sobre el pecho. Durante la tercera centuria, dichos ganchos fueron sustituidos por uno o dos pectorales repujados con idéntica función.

La lorica hamata presenta una serie de ventajas y desventajas ante el combate. L as anillas proporcionan una excelente defensa contra los cortes y también son eficaces contra los golpes de espada, pues son armaduras muy flexibles capaces de absorber las estocadas. Sin embargo se muestran vulnerables ante las flechas, cuyas puntas podían penetrar a una profundidad letal para el soldado (M assey, 1994: 36-37). Entre sus desventajas se encuentra el tiempo de elaboración y el peso excesivo (Simkins, 1994b: 18). Como ventajas tenemos su sencillo sistema de construcción, el cual facilita la reparación de las partes dañadas. Este hecho justifica el escaso número de corazas completas o significativamente intactas conservadas, ya que cuando se dañan sólo es necesario repararlas sustituyendo las anillas rotas. Esta peculiaridad propició el reaprovechamiento constante de los especimenes, que pasaban de una generación a otra de soldados, aspecto que justifica su escasa presencia en los establecimientos militares de larga duración, como es el caso de los fuertes hispanos y sobre todo el de L eón.

Existen pocos restos en Hispania concluyentemente atribuibles a esta categoría de corazas, pues ante el hallazgo de anillas sueltas es imposible discernir su pertenencia al mundo

certamen, donde figuraban con el número 1123 (B ello Diéguez, 1991: 57-58). A gradecemos a Tomás Vega la información dada al respecto. Posteriormente se han mencionado en diversas publicaciones, aunque sin ilustrarlas (C aamaño, 2000: 285).

13 Allason-Jones, L.: Part 1: The metalwork. Capítulo 11, p. 45 de la obra digital: http://www.barbicanra.co.uk/Piercebridge.htm. Consultado el 23/11/08. 
de las armaduras o a otro tipo de objetos. Es por ello que no recogeremos tales descubrimientos, aunque en ocasiones podamos sospechar que nos encontramos ante el testimonio de reparaciones efectuadas en tales lorigas, como por ejemplo el fragmento de una pequeña anilla de bronce recubierta de hierro, encontrada en un contexto de la legio VII en el edificio B otines de L eón.

Los primeros indicios de estas cotas de malla datan de época republicana, así los procedentes de Renieblas (Soria) (Schulten, 1929: Iám. 26, 20), si bien son más comunes a partir del Imperio. Durante los dos primeros siglos de nuestra Era, podemos rastrear el uso de estas armaduras gracias a los ganchos curvados que se ubicaban en el centro del pecho y que servían para abrochar las hombreras. Dichos garfios desaparecen completamente del registro arqueológico de todo el Imperio durante la segunda mitad del siglo II.

La morfología habitual de estos ganchos es una pronunciada «s» rematada en uno de sus extremos por un apéndice perforado y en el otro por una cabeza zoomórfica. Este modelo está muy extendido por todo el orbe militar romano, documentándose en Hispania mediante una pieza inédita procedente del campamento de Petavonium (Zamora) (fig. 3, 1) 14. Entre las piezas análogas podemos citar las descubiertas en Vindonissa (Suiza) (Unz y Deschler-Erb, 1997: lám. 35), si bien los paral elos más cercanos, virtualmente idénticos, proceden de la base augustea de Dangstetten (A lemania) (Fingerlin, 1986: 180,1; 1998: 1337, 6). L as similitudes morfológicas entre los ejemplares de Rosinos de Vidriales y Dangstetten, los cuales incluso comparten la misma sintaxis decorativa, nos inducen a pensar que el ejemplar zamorano debe estar relacionado con los primeros momentos del asentamiento de la legio $X$ en el lugar.

M ucho menos frecuente es otra modalidad de gancho en la que el apéndice figurado se sustituye por una superficie explayada bilobulada con sendas perforaciones. Con este formato tenemos un ejemplar hispano, también inédito, procedente de Puente Castro (L eón) (fig. 2, 3), vinculado a un estrato fechado entre la segunda mitad del siglo I y la primera mitad del siglo II. Entre los escasos especímenes conocidos en el resto del Imperio, destaca la concentración de ejemplares en G ran B retaña (B arnard Castle, L ongthorpe y Chester) (Bishop, 1989: 11, fig. $3)$, con los cuales guarda evidentes paral elismos el gancho leonés.

\section{PECTORALES REPUJADOS}

Hemos escindido en un capítulo aparte estas piezas ya que se usaron sobre distintos tipos de armaduras. Principalmente se emplearon en las armaduras de escamas, facilitando la introducción de la cabeza por la abertura que flanqueaban, abertura que se cerraba mediante el solapamiento de una placa sobre otra. No obstante se utilizaron pectorales idénticos en las cotas de malla, como se desprende del descubrimiento de B ertoldsheim (Garbsch, 1984: 239$53)$; incluso fueron adoptados en una variante minoritaria de las armaduras segmentadas, la denominada «A Iba-Iulia» (B ishop, 2002: 62-65).

Su rasgo más específico, aparte de su forma, es que cuentan con una rica decoración repujada, característica que motivó su inclusión entre los ornamentos propios de la hippika gimnasia, si bien Petculescu (1990: 846-849) demostró que dichas placas tuvieron un uso plenamente funcional, siendo empleadas en el campo de batalla tanto por soldados de infantería como de caballería. Sobre la cronología, estas piezas comienzan a documentarse durante la segunda mitad del siglo II, si bien son más características de la centuria siguiente, estableciéndose su fecha final de uso en torno al tercer cuarto de esta última centuria (Petculescu, 197475: 86-87). A nuestro juicio tal temporalidad habría que adelantarla hasta finales del siglo III 0

14 Ver nota 7. 
los primeros albores del cuarto, tal y como señalan los hallazgos hispanos y algunos indicios britanos (A urrecoechea, 2007: 173).

En H ispania únicamente conocemos un par de ejemplares, ambos localizados en el entorno del campamento de la legio VII gemina en León. U no de ellos se localizó en las excavaciones de Legio, concretamente en el vertedero de Santa M arina, en un contexto datado en los inicios del siglo IV d.C. (A urrecoechea y García, 2006: 112-119). La sintaxis decorativa se articula en torno a un águila, motivo repetido hasta la saciedad en estos petos; y una representación esquemática de las armas de un soldado. L os lateral es están ocupados por rectángulos alargados con sendas perforaciones, las cual es sirvieron para asir la pieza a la armadura y abrocharla con su placa gemela. De los elementos de sujeción sólo se conserva uno, consistente en un aplique con forma de busto masculino ( ¿M arte?), rematado por un apéndice en forma de «T» (fig. 2, 1). Entre los pectoral es similares mencionaremos los descubiertos en Pfünz (Alemania) (Garbsch, 1978: taf. 9, 7) y Zugmantel (Alemania) (Robinson, 1975: 161).

De Puente Castro (León) procede otro fragmento de pectoral, hasta ahora inédito (fig. 2, 2). A unque lo conservado es bastante exiguo, sin embargo es suficiente como para reconstruir el motivo que decoraba el registro inferior, consistente en un escudo agallonado. Por su estructura y ornamentación podemos encuadrarlo dentro de la categoría de petos más antiguos, datados en la segunda centuria, cronología que coincide con el ambiente arqueológico del que procede, pues fue descubierto en un nivel fechado entre la segunda mitad del siglo II y la primera mitad de la centuria siguiente. Piezas similares han sido encontradas en Pfünz, M undelsheim (A lemania) (Garbsch, 1978: taf. 9, 11), Porolissum y Buciumi (Rumania) (Petculescu, 1974-75: 81-83, fig. 1-2).

A unque no podemos aventurar el tipo de corazas al que estuvieron asociados los pectorales hispanos conocidos, reseñaremos que ambos aparecieron en contextos donde fueron localizados vestigios de armaduras. Concretamente el pectoral de L egio apareció en el mismo estrato arqueológico donde se documentaron fragmentos de lorica segmentata, mientras que el de Puente Castro proviene de la capa donde fueron descubiertos los restos de lorica squamata descritos anteriormente.

\section{PROTECTORES PARCIALES PARA BRAZOS Y PIERNAS}

La protección de las extremidades durante el combate fue una constante preocupación, sobre todo porque cualquier daño infligido en ellas mermaba la capacidad ofensiva del soldado, lo que fomentó el empleo de protectores parciales, como los guardabrazos, los guardamuslos o las espinilleras. Estos el ementos se fabricaron no solo en metal sino también en cuero, lo que dificulta rastrear el uso real de estas piezas, pues raramente han llegado hasta nosotros los ejemplares fabricados en material orgánico.

El brazo fue objeto de una especial atención mediante el diseño de un protector formado por láminas de hierro o cobre/bronce imbricadas entre sí, denominado manica. Estos guardabrazos se utilizaban individualmente, cubriendo uno sólo de los miembros, aquel que empuñaba la espada, pues el otro brazo quedaba resguardado por el escudo. Se han documentado escasos testimonios arqueológicos de estos objetos, entre los que cabe citar los descubiertos en Carnuntum (A ustria) (Groller, 1901: 1115-1184), U Ipia Traiana Sarmizegetusa (Rumanía) ${ }^{15}$, Newstead (Robinson, 1975: 184-186) y Carlisle (M cCarthy et alii, 2001). L a escasez de restos resalta la importancia de hallazgos como el documentado en León, máxime si tenemos en

15 Queremos agradecer a M ike B ishop la información ofrecida sobre las piezas rumanas. 
cuenta que el ejemplar hispano se encuentra casi completo. A pareció en un contexto datado en el tercer cuarto del siglo III, durante los trabajos efectuados en Santa M arina (A urrecoechea et alii, 2008). Entre las singularidades que presenta está la de haber preservado parte del sistema de abrochado, algo excepcional que sólo cuenta con paralel os en el reciente descubrimiento de Carlisle, así como conservar completa la estructura que protegía el puño y dorso de la mano (fig. 3, 2).

Respecto a los protectores para las piernas, Polibio (6.23) sugiere que sólo se usaba una espinillera ubicada en la pierna izquierda, dado que la otra se defendía con el escudo. Entre las grebas documentadas en contextos castrenses se encuentran aquellas descubiertas en $\mathrm{K}$ ünzing (A lemania) (Garbsch, 1978: lám. 3). No conocemos en Hispania testimonios de espinilleras vinculadas indiscutiblemente al mundo militar, si bien se han documentado piezas que podrían ser candidatas, aunque debemos tener en cuenta que también podrían estar relacionadas con los juegos gladiatorios; así la hallada en Pollentia (Mallorca) (A A.VV., 1990: № 37), o el ejemplar de M érida (Nogales, 2000: 64).

\section{EQUIPO EQUINO}

Las armaduras equinas tuvieron dos funciones distintas: la protección facial durante los encuentros deportivos y la protección integral durante la batalla. El equipo para la hippika gimnasia de los siglos I-II cubría solamente la cabeza del caballo, mediante una máscara de cuero del tipo de la aparecida en N ewstead (Curle, 1911: lám. 21), complementada por unos protectores oculares de metal. Durante el siglo III se impone un nuevo modelo, consistente en máscaras formadas por tres placas metálicas charneladas, como la hallada en Straubing (A lemania) (Garbsch, 1978: lám. 4-5). Otra modalidad de la tercera centuria son unas guardas curvadas que cubrían solamente los ojos (Garbsch, 1978: lám. 6).

Fue en la P ars Orientalis del Imperio, y sobre todo durante el B ajo Imperio, cuando se hizo perentoria la necesidad de preservar de forma integral al caballo. Surgen entonces las unidades de caballería pesada denominadas catafractarii y clibanarii (Speidel, 1984). L as circunstancias bélicas impusieron las armaduras equinas, necesarias para poder combatir contra los arqueros enemigos que protagonizaban buena parte de las campañas en el este. Respecto a su fabricación, estaban realizadas con escamas, tal y como conocemos gracias a los ejemplares de Dura Europos (Siria) (J ames. 2004: 259, lám. 13). Discernir los restos de estas armaduras cuando solamente aparecen escamas suel tas es una labor infructuosa, por lo que no podemos asegurar si alguna de las escamas documentadas en $\mathrm{H}$ ispania pudieron proteger a un équido. No obstante, creemos que esta interpretación es muy improbable, ya que esta impedimenta es más típica de los escenarios de guerra orientales.

Por el contrario si contamos con un testimonio de la hippika gimnasia, concretamente el par de anteojeras descubiertas en el «Cuartel II» de Herrera de Pisuerga (fig. 1, 16), cuyo contexto arqueológico las fecha en época augustea (Pérez, 1995: 53-68). Dicha datación convierte al ejemplar hispano en el más antiguo conocido en todo el Imperio, haciendo plausible la teoría de su utilización en el combate real y no sólo como el emento de parada militar, debido a su proximidad temporal con las Guerras Cántabras y al análisis de un ejercicio militar denominado «Cantabricus ímpetus» (Pérez, 1995: 59). A unque los protectores oculares son mucho más habituales durante las centurias posteriores, sin embargo, conocemos otros ejemplares tempranos del siglo I, como por ejemplo, los de Mainz (A lemania) y Pompeya (Italia) (Garbsch, 1978: S3, lám. 45, 5-6 y S11, lám. 47, 1). 


\section{FABRICACIÓN}

Si bien la producción de equipo militar no puede ser tratada de forma homogénea, la fabricación de enseres militares en gran parte de los fuertes romanos de los siglos II y III, o en las áreas civiles próximas, es una realidad ampliamente atestiguada (A urrecoechea, 2006). Se trataba de una necesidad, que en el caso concreto de las armaduras es aún más evidente. Estos protectores debían fabricarse según la talla y medidas de cada soldado, y aunque debieron existir porciones estándar para determinadas partes anatómicas, éstas debían adaptarse a cada individuo. A I mismo tiempo, nos encontramos ante un tipo de impedimenta proclive al deterioro, por lo que era indispensable la reparación constante de los desperfectos. EI panorama que esbozamos justifica por si solo la confección y mantenimiento de armaduras en los establecimientos militares hispanos, pero además contamos con datos arqueológicos que avalan tal suposición.

Tenemos la total certeza de que es así en Legio, campamento en el que se ha atestiguado una intensa actividad productiva relacionada con los enseres metálicos de índole militar. La constatación de varios talleres legionarios en León, incluido uno de ellos especializado en la realización de loricae segmentatae (A urrecoechea, 2006: 309-328), así como el hallazgo de material es desechados en las sucesivas remodel aciones del campamento (A urrecoechea, 2006: 329-331), confirmarían tal aseveración.

Del campamento establecido en Herrera de Pisuerga conocemos aún solo datos parciales sobre su actividad metalúrgica, aunque parece que buena parte de ella se destinó a la impedimenta militar, entre la que podría incluirse a las armaduras, si bien no existe constancia implícita de ello. Podemos mencionar, a título de ejemplo, la aparición de un taller en el yacimiento del «Cuartel I» asignable al cuerpo auxiliar ala Parthorum (Pérez e Illarregui, 2006: 111-133, fig. 17).

\section{CONCLUSIONES}

El crecimiento exponencial de la información, debido a las intensas campañas de excavación efectuadas en los últimos años sobre los principales enclaves militares de Hispania, hace que nos encontremos en una fase de conocimiento meramente especulativa. Cualquier teoría que se postule deberá ser revisada en el futuro, a tenor de los nuevos descubrimientos que se produzcan. Entre las razones que limitan el establecimiento de conclusiones definitivas destaca:

a) La parcialidad de la información, ya que no contamos con hallazgos de armaduras completas o fragmentos significativos como para definir la estructura íntegra de la coraza. L a gran mayoría de los hallazgos son piezas de desecho destinadas al reciclaje. El estado fragmentario de los restos condiciona las conclusiones sobre tipologías y modelos.

b) Carecemos de datos como para establecer una distribución regional de los hallazgos, ya que nuestro conocimiento está sesgado por la abundancia de información sobre yacimi entos concretos, sobre todo aquellos que se desarrollaron en el entorno de L eón. Este desequilibrio es fruto del desigual tratamiento dado a los materiales metálicos aparecidos en cada campamento, pues no suelen publicarse de forma conjunta o sis- 
temática, sino de forma aislada ${ }^{16}$. Un caso significativo es Herrera de Pisuerga, yacimiento para el que repetidamente se señala la existencia de restos de cotas de malla, Iorica segmentata y placas de lorica squamata, si bien aún estamos a la espera de su publicación definitiva.

c) Otro problema deriva de la mala interpretación de los hallazgos, pues a menudo encontramos publicadas piezas de muy dudosa filiación militar catalogadas como restos de armaduras, caso de los hallazgos de A txa (Vitoria) ${ }^{17} 0$ algunos de los materiales de Conimbriga (Portugal) ${ }^{18}$.

d) La cronología de los hallazgos es otro factor que limita el establecimiento de teorías generales, pues el reciclaje constante del metal que se efectuaba en los campamentos romanos hace que los descubrimientos se concentren en los momentos de abandono de los yacimientos o en los periodos de remodelaciones rápidas ante situaciones de crisis. Es por ello que el siglo III se encuentra sobrevalorado en el registro arqueológico, mientras que, por ejemplo, apenas conocemos datos de la segunda centuria.

A un teniendo en cuenta las premisas anteriores, podemos establecer una serie de conclusiones.

Desde el punto de vista temporal, podemos atisbar que el ejército hispano contó durante todo el Imperio con la armadura como parte integrante de su uniforme habitual, a pesar de no ser una provincia fronteriza. Los testimonios, repartidos desde el siglo I al V, demuestran que a pesar de que nuestro ejército se encontraba en una zona pacificada siempre estuvo preparado para la lucha.

Respecto a las loricae segmentatae los hallazgos hispanos ofrecen la datación más avanzada de todas las conocidas en el Imperio Romano, poniendo en tela de juicio la datación que tradicional mente se asignaba a las distintas variantes de estas corazas. A sí el tipo «K alkriese» parece prolongarse hasta la segunda mitad del siglo I, la categoría «Corbridge» perduró hasta el último cuarto del siglo III, mientras que la clase «N ewstead» aún estaba vigente durante la primera mitad del siglo IV (A urrecoechea, 2007: 177). L o mismo cabe decir de los petos con decoración repujada, que en Hispania alcanzan el Tardo Imperio. Los testimonios de armaduras segmentadas de la península ibérica también cuestionan uno de los principios básicos más extendidos sobre estas corazas: Ia teoría de que las distintas variantes no son más que evoluciones de un tipo que deriva en otro, por lo que se sucederían en el tiempo sin apenas solapamiento temporal (A urrecoechea, 2007: 178).

Los descubrimientos avalan el uso de armaduras a finales del siglo IV o inicios del V, desmintiendo así la teoría que preconiza la práctica desaparición de la coraza metálica, especialmente entre los cuerpos de infantería, durante el Bajo Imperio. Dicha teoría, asentada sobre la iconografía tardorromana, donde se muestran habitualmente soldados sin lorigas; y la aseveración que hizo Vegetius (1.20), quien nos dice que los cascos y las armaduras eran objetos raros dentro de la infantería de Graciano (367-383), ya fue desmentida por Bishop y

16 A unque existen notables excepciones, así Aquis Q uerquennis (Orense). Sin embargo en tal enclave no se han documentado restos de armaduras, como sabemos por Tomás Vega, a quien agradecemos tal información.

17 Nos referimos a dos tachuel as publicadas como accesorios de armaduras segmentadas. Sin embargo, las dimensiones de los vástagos aguzados (al rededor de $25 \mathrm{~mm}$ ) y la tipología de las piezas, indican que nos encontramos ante remaches ornamentales de cajas o muebles. También desestimamos una presunta escama de lorica squamata, debido a las características de sus perforaciones (Gil, 1995: 330, nos. 153-154 y 327, № 1926). Tampoco incluimos la escama hallada en La Iglesia (L aguardia, Álava) por tratarse de un material de prospección de dudosa cronología (Gil, 1990: 161, fig. 4, 5).

18 A unque $C$ onimbriga cuenta con objetos militares, no creemos que ninguno de los publicados como armaduras lo sean. A sí el hipotético gancho de segmentata es en realidad la mitad de un enganche o conector de atalaje, y la supuesta bisagra es más propia de los cierres de baúles y cajas romanas (Alarcão, 1979: 98, no 81 y 99, ํㅡ 93). 
Coulston $(1993,167)$. L os hallazgos producidos en el entorno del campamento de L eón son la confirmación arqueológica de que las armaduras metálicas no sólo seguían usándose, sino que su utilización distaba de ser residual, ya que en Hispania destacan porcentualmente el número de ejemplares datados en época tardía. Precisamente las corazas «N ewstead» se difundieron en los asentamientos cercanos a Legio durante la etapa bajoimperial, como demuestran los descubrimientos de Puente Castro, Lancia y A storga.

En lo tocante al lugar de fabricación, a tenor de lo que manifiesta L eón, podemos intuir la autarquía de los campamentos hispanos, ya que en cada enclave se fabricarían las armaduras «a medida» para cada soldado.

En cuanto a la dispersión, podemos afirmar que los vestigios de lorigas aparecen claramente vinculados con establecimientos del mundo militar (campamentos y canabae). A diferencia de lo que ocurre con otros elementos de la indumentaria castrense que aparecen en contextos civiles, no se conocen restos de armaduras procedentes de núcleos urbanos o rurales, lo que implica que estas piezas estaban fuera del comercio que las unidades militares solían hacer de sus excedentes ${ }^{19}$. Tampoco parece que los soldados licenciados tuvieran por costumbre llevarse las armaduras como «recuerdo» de su paso por el ejército, tal y como hacían con otros objetos más personales, como los cinturones ${ }^{20}$.

A cerca de los modelos constatados en Hispania, los testimonios de armaduras segmentadas descuellan por su abundancia frente a otros tipos mucho menos representados (como la lorica hamata). En ocasiones se ha señalado que la mayor presencia de lorigas segmentadas en el registro arqueológico se debía a que se deterioraban más fácilmente que el resto (B ishop, 1998: 10-14), si bien pensamos que ello no justifica su profusión en los yacimientos hispanos, máxime si tenemos en cuenta que hay casos constatados en otras partes del Imperio, como Zeugma (Feugère, 2006: 93), donde las loricae segmentatae son una minoría. En el campamento de L eón y enclaves circundantes, los restos de corazas segmentadas suponen más de un $95 \%$ del total de las armaduras encontradas, cifra que creemos muy significativa, ya que para calcular dicha proporción no se ha tenido en cuenta el número de fragmentos, sino el número de unidades estratigráficas donde aparecen. A demás, concretamente las armaduras «N ewstead» apenas se documentan en la Europa M eridional ${ }^{21}$, Io que confiere un valor excepcional a los hallazgos hispanos.

Algunos investigadores han teorizado sobre el uso restringido de cada tipo de armadura, vinculándolo a estamentos militares concretos, así las segmentadas serían propias de los legionarios, la lorica squamata característica de los oficiales y las cotas de malla estarían reservadas a los oficiales. Del mismo modo, se ha señalado que las loricae squamatae estarían relacionadas exclusivamente con los cuerpos auxiliares y no con los legionarios (F ernández, 2005: 210). Dichas hipótesis no serían corroboradas por los testimonios hispanos, ya que en la mayoría de los yacimientos conviven todas las categorías mencionadas, independientemente de las unidades que los ocupen. Ello demostraría la inviabilidad de relacionar armaduras con unidades concretas, al menos en lo que se refiere a general izaciones válidas para todo el territorio del Imperio. El ejército romano no suponía una entidad homogénea y uniformada al estilo de las tropas modernas, debiendo estudiarse cada zona concreta en periodos determinados de su historia.

19 En zonas como Germania se ha demostrado que las legiones vendían los excedentes de sus fabricae, sobre todo los objetos metálicos empleados en los atalajes equinos.

20 Las causas que justifican el hallazgo de piezas militares en contextos civiles las hemos tratado en trabajos anteriores (A urrecoechea, 1995/96. 2001).

21 A sí, en todo el sur de la Galia sólo se conoce una anilla suelta encontrada en Clermont-l'H érault (Feugère, 2002: fig. 10, $\mathrm{n} 035$ ). 


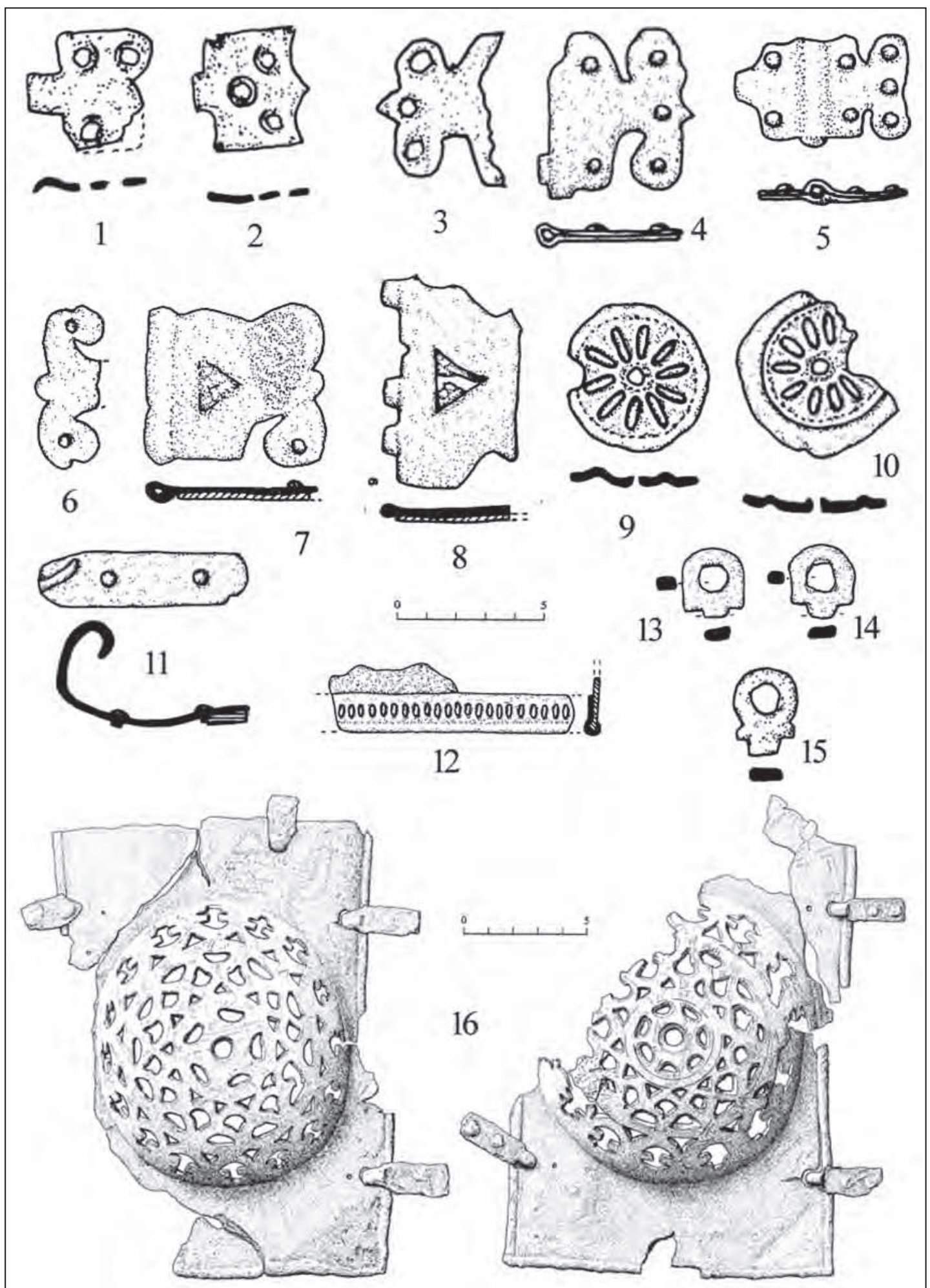

Figura 1. Bisagras de A storga: (1 y 3) Calvo Sotelo y (2) Padre Blanco. Accesorios de León: (4 a 15) Plaza del Conde Luna. A nteojera de Herrera de Pisuerga según Pérez (16). 


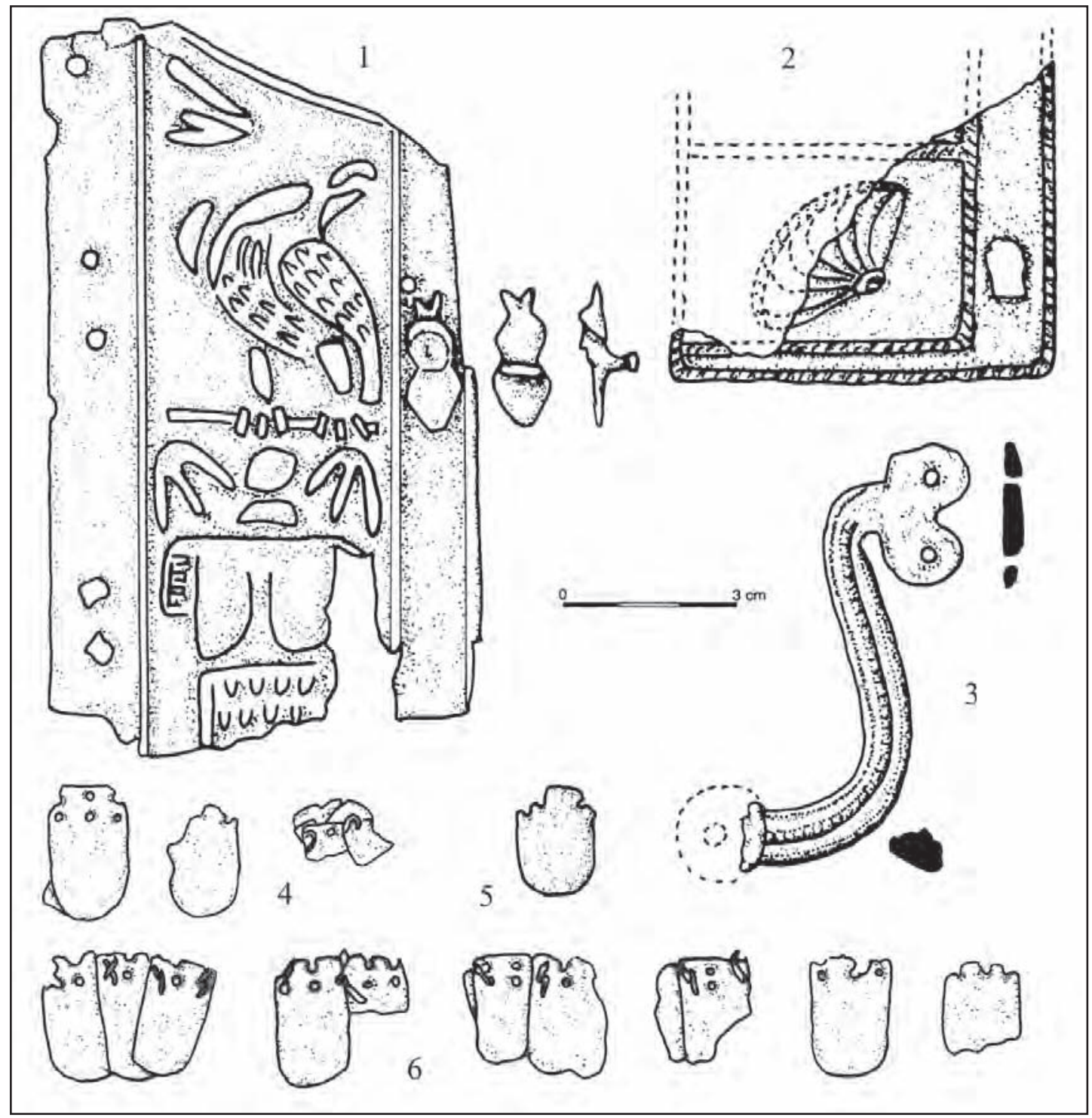

Figura 2. Pectorales: (1) Santa M arina/San A lbito y (2) Puente Castro. Gancho: (3) Puente Castro. Escamas: (4) Plaza del Conde L una, (5) Santa M arina) y (6) Puente Castro. 


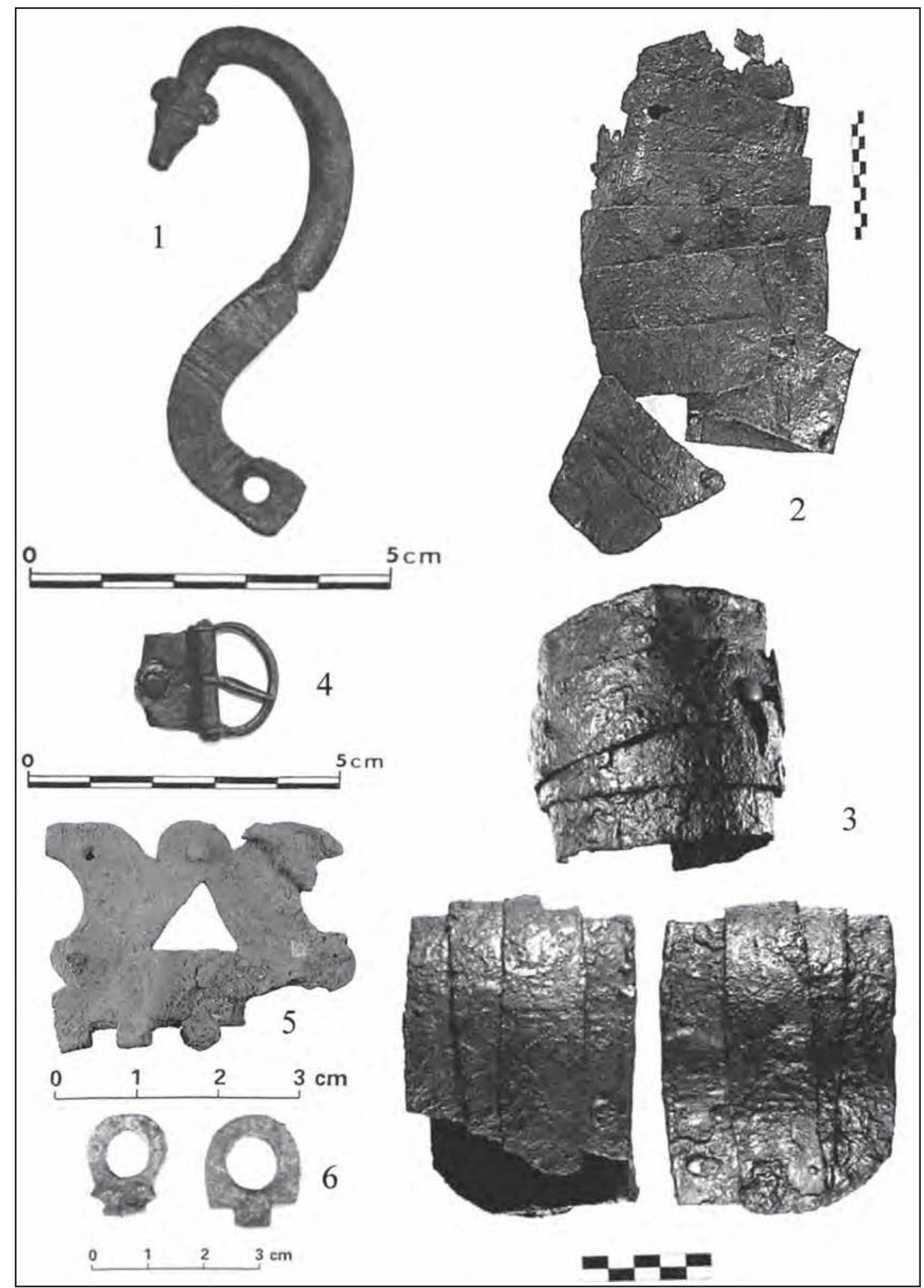

Figura 3. Gancho y hebilla de Rosinos de Vidriales, F oto Junta de Castilla y L eón, M useo de Zamora: (1 y 4). M anica de León: (2) Puerta Castillo/Santa M arina. A rmaduras de León: (3) La Praviana, (5) Plaza del Conde Luna) y (6) Cid/Ordoño. 


\section{BIBLIOGRAFÍA}

A A.V V. (1990): Los bronces romanos en España. M adrid.

A larcão, J. (1979): F ouilles de Conímbriga. Tomo VII. Trouvailles diverses- Conclusions Générales, París.

Álvarez, J. C., Rodríguez, P. y M artínez, N. (2000-2001): «Instrumental médico procedente de la excavación del yacimiento de época romana de la carretera del cementerio. Puente Castro. León: Una aproximación». Lancia, 4: 141-158.

A urrecoechea Fernández, J. (1995-1996): «L as guarniciones de cinturón y atalaje de tipología militar en la Hispania Romana, a tenor de los bronces hallados en la M eseta Sur». Estudios de Prehistoria y Arqueología Madrileñas, 10: 49-100.

A urrecoechea Fernández, J. (2001): «A ccesorios metálicos de correas de cinturones militares al toimperiales hallados en Hispania». AEspA, 74: 291-302.

A urrecoechea Fernández, J. (2006): «Talleres dedicados a la producción de equipo militar en los campamentos romanos de León, con especial referencia a los restos de lorica segmentata», A . M orillo (ed.), Arqueología militar romana en Hispania II: producción y abastecimiento en el ámbito militar. León: 309-334.

A urrecoechea Fernández, J. (2007): «Las armaduras segmentadas (loricae segmentatae) en los yacimientos romanos de la provincia de L eón: un estudio de conjunto». AE spA, 80: 153-182.

A urrecoechea Fernández, J., Fernández I báñez, C., García M arcos, V. y M orillo, A. (2008): «U n protector laminado de brazo (manica) procedente del campamento de la legio VII Gemina en León». AEspA, 81: 255-264.

A urrecoechea, Fernández, J. y García, V. (2006): «Un pectoral de armadura con decoración repujada, hallado en el campamento de la Legio VII en L eón». Sautuola, 12: 112-119.

Bello DiéGuez, J. M. (1991): «La integración de La Coruña en el espacio romano». Ciudad y Torre. Roma y la Ilustración en La Coruña. La Coruña: 51-73.

B eltrán L loris, M . (1998): «M useo de Zaragoza. Colonia C elsa. Catálogo M onográfico». Boletín del M useo de Zaragoza, 14: 5-172.

Bishop, M. C. (1989): «A new mail hook». Arma, 1: 11-12.

Bishop, M. C. (1998): «The development of lorica segmentata: recent advances and old work re-assessed». Arma, 10: 10-14.

Bishop, M . C. (2002): Lorica segmentata, Vol. I: A handbook of articulated roman plate armour, B raemar.

Bishop, M. C. y Coulston, J. C. (1993): Roman M ilitary E quipment from the Punic Wars to the F all of Rome, Londres.

Caamaño Gesto, J. M . (1983): "A portaciones al estudio de la Cohors I Celtíberorum: una inscripción militar hallada en el campamento romano de Cidadela (Sobrado dos M onxes, Coruña)". Brigantium, 4: 61-71.

Caamaño Gesto, J. M . (2000): «Evidencias materiales en el campamento romano de Cidadela (Sobrado dos M onxes, A Coruña», Actas do 30 Congresso de Arqueologia Peninsular, Vol. I: Arqueologia peninsular. historia, teoria e pratica. Porto: 281-289.

Curle, J. (1911): A Roman Frontier Post and its People. The F ort at N ewstead. Glasgow.

Down, A . (1989): Chichester Excavations 6. Chichester.

Driel-M urray, C. Van (ed.) (1989a): Roman M ilitary Equipment: the Sources of Evidence. Proceedings of the Fifth Roman M ilitary E quipment Conference. Oxford, BA R International Series, 476.

Driel-M urray, C. Van (1989b): «The Vindolanda chamfrons and miscellaneous items of leather horse gear», C. van Driel-M urray (ed.), Roman M ilitary Equipment: the Sources of Evidence. Proceedings of the Fifth Roman M ilitary Equipment Conference. Oxford. BA R International Series, 476.

Fernández Ibáñez, C. (2005): «M etal istería militar romana en el norte de la península ibérica durante los períodos republicano y altoimperial». III Coloquio Internacional de Arqueología en Gijón III Coloquio Internacional de Arqueología en Gijón: U nidad y diversidad en el Arco Atlántico en época romana. Oxford, BAR International Series, 1371: 203-228.

Fernández Ibáñez, C. (1994): «Un castrum legionis en Herrera de Pisuerga (Palencia)». 0 Rumor da Fraga, 7: 11-14. 
Feugère, M . (2002): «M ilitaria de Gaule méridionale, 19: Le mobilier militaire romain dans le département de I'H érault (F)». Gladius, XXII: 73-126.

Feugère, M . (2006): «R oman militaria from Zeugma», International Symposium on Zeugma; from past to future, Gaziantep.

Filloy, I. y Gil, E. (2000): La romanización en Álava. Catálogo de la exposición permanente sobre Alava en época romana del Museo Arqueológico de Álava. Vitoria.

Fingerlin, G. (1986): Dangstetten I. Katalog der F unde (F undstelle 1 bis 603). Forschungen und Berichte zur Vor- und Frühgeschichte in B aden-W ürttemberg 22, Stuttgart.

Fingerlin, G. (1998): D angstetten II. Katalog der F unde (F undstelle 604 bis 1358). Stuttgart, Forschungen und B erichte zur Vor- und Frühgeschichte in B aden-Württemberg, 69.

Garbsch, J. (1978): Römische Paraderüstungen, M unich.

Garbsch, J. (1984): «E in römisches Paradekettenhemd von B ertoldsheim, L dkr. N euburg-Schrobenhausen». Neuburger Kollektaneenblatt, 136: 239-53.

García-B ellido, M. P. (coord.) (2006): Los campamentos romanos en Hispania (27 a. C.-192 d. C.). EI abastecimiento de moneda. A nejos de G ladius, 9, M adrid.

García Díez, F. (1999): «A Igunas piezas metálicas romanas de Herrera de Pisuerga». Papeles Herrerenses, 2: 13-31.

Gil, E. (1990): «A Igunos el ementos metálicos de equipo militar romano en Á lava». Estudios de Arqueología Alavesa, 17: 145-165.

Gil, E. (1995): Atxa. M emoria de las excavaciones arqueológicas 1982-1988. Vitoria, M emorias de yacimientos alaveses.

Gil, E. (1997): «El instrumental metálico de época romana en Á lava: testimonio de actividades domésticas y profesional es». Isturitz, 9: 535-563.

González, M. L. y Vidal, J. (2004): «La Legio X G émina y Astúrica (A storga - León)», C. Pérez-González et E. Illaregui (coord.), Arqueología militar romana en Europa (Segovia, 3-14 de julio de 2001). Segovia, Universidad Internacional SEK : 207-218.

Groller, M. Von (1901): «Das Lager von Carnuntum». Der Römische Limes in Österreich, 2: 15-84.

$J$ ames, S. (2004): The excavations at D ura-E uropos conducted by Yale U niversity and the F rench Academy of Inscriptions and Letters 1928 to 1937. Final Report VII: The arms and armour and other military equipment. Londres, The B ritish M useum Press.

M assey, D. (1994): «R oman A rchery Tested». M ilitary Illustrated: Past \& Present, 74: 36-38.

M cCarthy, M., Bishop, M. C. y Richardson, T. (2001): «Roman armour and metalworking at Carlisle, Cumbria, England». Antiquity, 75: 507-508.

M enéndez, A. R. (2006): «Una cuestión a debate: la lorica segmentata en las fronteras orientales del imperio romano». Gladius, XXVI: 105-117.

M orillo, A. y García, V. (2006): "Legio (León): cronologías estratigráficas". M. P. García-B ellido (coord.), Los campamentos romanos en Hispania (27 a. C.-192 d. C.). El abastecimiento de moneda. A nejos de G ladius, 9. M adrid: 244-257.

N ogales Basarrate, T. (2000): Espectáculos en Augusta Emérita. M onografías Emeritenses, 5. Badajoz.

Pérez González, C. (1995): «Una testera militar de caballo en bronce de época augustea en España». Anuario de la Univer sidad Internacional SEK, 1: 53-68.

Pérez González, C. (1996): "A sentamientos militares en Herrera de Pisuerga", C. Fernández Ochoa (ed.), C oloquio Internacional sobre los F inisterres Atlánticos en la Antigüedad. É poca Prerromana y Romana (Gijón, 1995), Gijón: 91-102.

Pérez, González, C. e Illarregui, E. (2006): «Producciones militares en el campamento de la legio IIII Macedonica en Herrera de Pisuerga», A . M orillo (ed.), Arqueología militar romana en H ispania II: producción y abastecimiento en el ámbito militar, León: 111-133.

Petculescu, L. (1974-75): «Garnituri de armura romane din Dacia». Studii si materiale de muzeografie si istorie militara, 7-8: 79-88.

Petculescu, L. (1990): «Contributions to R oman decorated helmets and breastplates from Dacia», Akten des 14. Internationalen Limeskongresses 1986 in Carnuntum. Viena: 843-854.

Peterson, D. (1992): The Roman Legions Recreated in Colour Photographs, Europa Militaria Special N 0.2 , L ondon.

Robinson, H.R. (1975): The Armour of Imperial Rome, Londres. 
Schulten, A . (1929): Numantia. Die Ergebnisse der Ausgrabungen 1905-1912. IV. Die Lager bei Renieblas, Munich.

Simkins, M . (1994a). The Roman Army from Caesar to Trajan, Hong Kong.

Simkins, M. (1994b): The Roman Army from Hadrian to Constantine, Hong K ong.

Speidel, M .P. (1984): «Catafractarii-clibanarii and the rise of the L ater R oman mailed caval ry. A gravestone from Claudiopolis in Bithynia». Epigraphica Anatolica, 4: 151-156.

Thomas, M.D. (2003): Lorica segmentata, Vol. II: A catalogue of finds. B raemar.

Unz, C.\& Deschler-Erb, E. (1997): Katalog der Militaria aus Vindonissa. Militärische F unde, P ferdegeschirr und J ochteile bis 1976. B rugg Veröffentlichungen der Gesellschaft Pro Vindonissa, 14.

Williams, A .R. (1977): «Roman arms and armour: a technical note». J ournal of Archaeological Science, 4: 77-87.

Recibido: 01/07/2009

A ceptado: 12/04/2010 